\title{
lodine I 124 Monoclonal Antibody 8H9
}

National Cancer Institute

\section{Source}

National Cancer Institute. Iodine I 124 Monoclonal Antibody 8H9. NCI Thesaurus. Code C99632.

A radioimmunoconjug ate consisting of the iodine I 124-radiolabeled murine IgG1 monoclonal antibody (MoAb) 8H9 directed against the cell surface glycoprotein CD276 (4lg-B7-H3) with potential for radioimaging using positron emission tomography (PET). Through convection enhanced delivery, iodine I 124 monoclonal antibody 8H9 binds to the 4Ig domain of CD276, in turn CD276 expressing tumor cells may be visualized upon PET imaging of the iodine I 124 moiety. CD276, a tumor associated antigen and member of the B7 family of co-stimulatory proteins, suppresses natural killer (NK) cell and cytotoxic T-lymhocyte activation; it is expressed on the cell membranes of a wide variety of tumors of neuroectodermal, mesenchymal and epithelial origin and its expression is associated with increased aggressiveness, poor prognosis and resistance. 